\title{
Osteopathic manipulative treatment and nutrition: An alternative approach to the irritable bowel syndrome
}

\author{
Luca Collebrusco ${ }^{1}$, Rita Lombardini ${ }^{2}$ \\ ${ }^{1}$ Rehabilitation Unit, National Health Service of Umbria, Perugia, Italy \\ ${ }^{2}$ Department of Clinical and Experimental Medicine, University of Perugia, Perugia, Italy; rlomba@unipg.it
}

Received 12 February 2013; revised 12 March 2013; accepted 10 April 2013

Copyright (C) 2013 Luca Collebrusco, Rita Lombardini. This is an open access article distributed under the Creative Commons Attribution License, which permits unrestricted use, distribution, and reproduction in any medium, provided the original work is properly cited.

\begin{abstract}
A simple treatment plan for manual therapists is presented based on current evidence-based literature, it is designed to lessen chronic pain and inflammation in the Irritable Bowel Syndrome (IBS). A chronic continuous or intermittent gastrointestinal tract dysfunction, IBS appears due to dysregulation of brain-gut-microbe communication. An overview of its management using Osteopathic Manipulative Treatment (OMT) is described. In IBS OMT focuses on the nervous and circulatory systems, spine, viscera, thoracic and pelvic diaphragms in order to restore homeostatic balance, normalize autonomic activity in the intestine, promote lymphatic flow and address somatic dysfunction. Lymphatic and venous congestion is treated by the Lymphatic Pump Techniques and stimulation of Chapman's Reflex Points. The food itself, food allergies and intolerance could contribute to symptom onset or even cause IBS. Furthermore the "microbiota" greatly impacts on the bi-directional braingut axis communication. This paper also provides appropriate dietary modifications for patients with IBS.
\end{abstract}

Keywords: Irritable Bowel Syndrome; Chronic Visceral Pain; Osteopathic Manipulative Treatment; Nutrition

\section{INTRODUCTION}

The present paper defines a simple treatment plan for manual therapists, which is designed to reduce chronic pain and inflammation in the Irritable Bowel Syndrome (IBS). We provide an overview of its management using Osteopathic Manipulative Treatment (OMT), dietary modification and nutritional supplementation, according to the current evidence-based literature.

A chronic continuous or intermittent gastrointestinal tract (GIT) dysfunction, IBS was defined by Rome Committee III on the basis of abdominal discomfort or pain (Table 1) [1]. IBS is found in twice as many females as males in $10 \%-15 \%$ of the population, affecting all adult age groups up to 50 [2]. Although its etiology and pathophysiology remain uncertain, IBS appears due to "brain-gut axis" dysregulation. The central nervous system communicates with the gut by mediating the sympathetic and parasympathetic autonomic nervous systems (SANS and PANS) which control enteric nervous system (ENS) function. Alterations in SANS, PANS and ENS were described in IBS patients $[3,4]$. Compared with normal subjects, they have an increased vagal response to rectal distension, decreased vagal response to sigmoid distension, a blunted adrenocorticotropic hormone response and lower plasma cortisol levels, suggesting abnormalities in the central control mechanisms that are involved in autonomic and neuroendocrinal response to visceral stimulation [5]. Central mechanisms include anxiety, depression and somatisation; peripheral dysfunction is characterized by gut motility and secretion changes and visceral hypersensitivity [6,7]. IBS is associated with structural changes in grey matter density involving key areas in attention, emotion regulation, pain inhibition and visceral information processing [8]. Proinflammatory cytokines such as IL-6, TNF- $\alpha$ and IL-8,

Table 1. Rome III criteria.

At least 3 months, with onset at least 6 months previously of recurrent abdominal pain or discomfort ${ }^{*}$ associated with 2 or more of the following:

- Improvement with defecation; and/or;

- Onset associated with a change in frequency of stool; and/or;

- Onset associated with a change in form (appearance) of stool.

*Discomfort means an uncomfortable sensation not described as pain. 
mast cells and pro-inflammatory polyunsatured fatty acid metabolites were increased [9-11]. Other potential causes of IBS are diet and enteric flora abnormalities leading to low-grade inflammation/immune activation [12].

Symptoms are diarrhea-predominant (IBS-D), constipation-predominant (IBS-C), or alternating diarrhea and constipation (IBS-DC) [13]. Other symptoms are bloating, early satiety, nausea, heartburn, feeling of incomplete emptying on defecation, borborygmi and flatulence. Migraine, back pain, dyspareunia and muscle pain indicate a central hyperalgesic mechanism [14]. Abdominal pain (Figure 1) is associated with significant morbidity, including depression, poor quality of life, and even inability to work. Patients with IBS were more likely than non-IBS sufferers to complain of problems relating to diet and eating out, concentration, long journeys, physical appearance and personal relationships. They had more sick days off work with considerable social and economic costs [15].

\section{OMT}

Over a century ago Andrew Taylor Still devised a system of disease prevention and treatment through mechanical manipulation known as OMT. He identified the musculoskeletal system as a key to health, hypothesizing that every illness was the result of an anatomical disorder associated with physiological discord, termed "Somatic Dysfunction" (SD). SD is "impaired or altered function of related components of the somatic framework; skeletal, arthroidal, myofascial and related vascular, lymphatic and neural elements" [16]. It contributes to the effect of organic pathology and is catalogued as a disease of musculoskeletal system (ICD-9, code 739). Primary SD is completely reversible when correctly diagnosed and treated with OMT. Secondary SD also responds to OMT but will recur unless the primary pathology is identified and treated [17]. SD is diagnosed by palpation procedures ("A.R.T.T." examination) [18] (Figure 2).

OMT consists of a range of direct, indirect, combined, fluid and reflex-based manual techniques (Figure 3) that are applied specifically to a joint or non-specifically to a body area [19]. Direct techniques apply thrust, impulse, muscle contraction, fascial loading, or passive range of motion. They engage the restrictive barrier and use an activating force to achieve the tissue response and correct the SD. Indirect, fluid, balancing, or reflex-based techniques do not engage the restrictive barrier. They use fascial massage, fascial and soft tissue loading or unloading, hydraulic pressures, respiration phases and cranial or postural adjustments [20].

\section{OMT for IBS}

In IBS OMT focuses on the nervous and circulatory

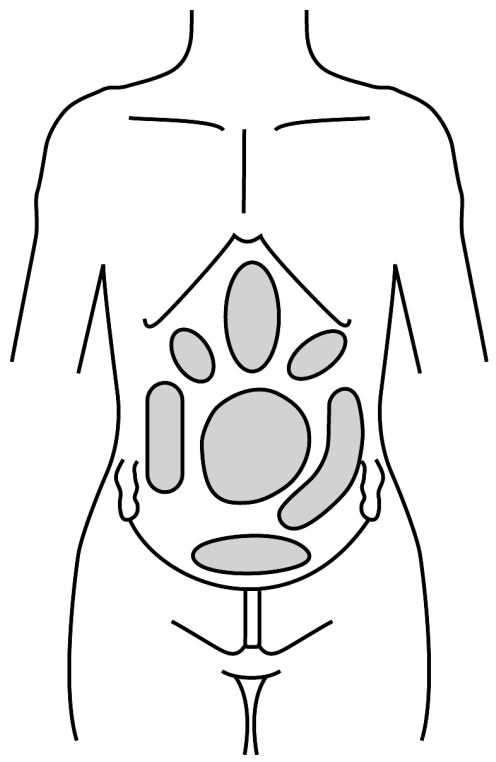

Figure 1. Areas of pain associated with IBS (modified from Stone 2007).

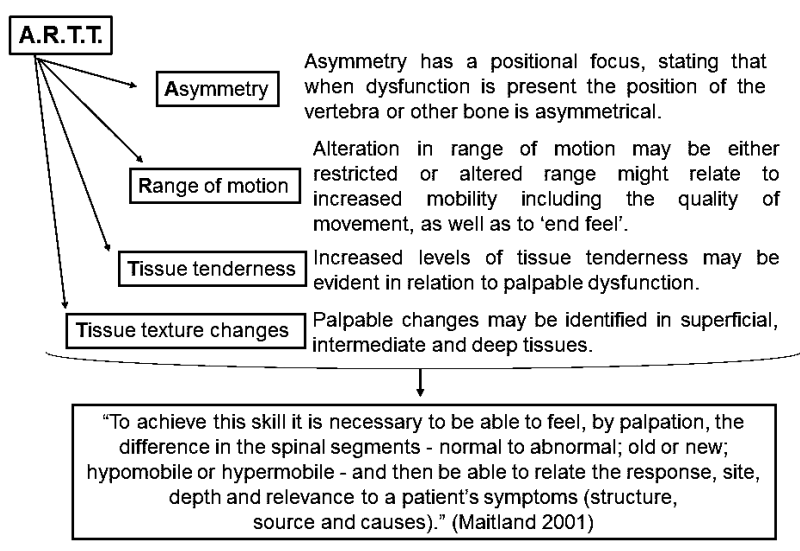

Figure 2. OMT palpation procedures or the ARTT technique.

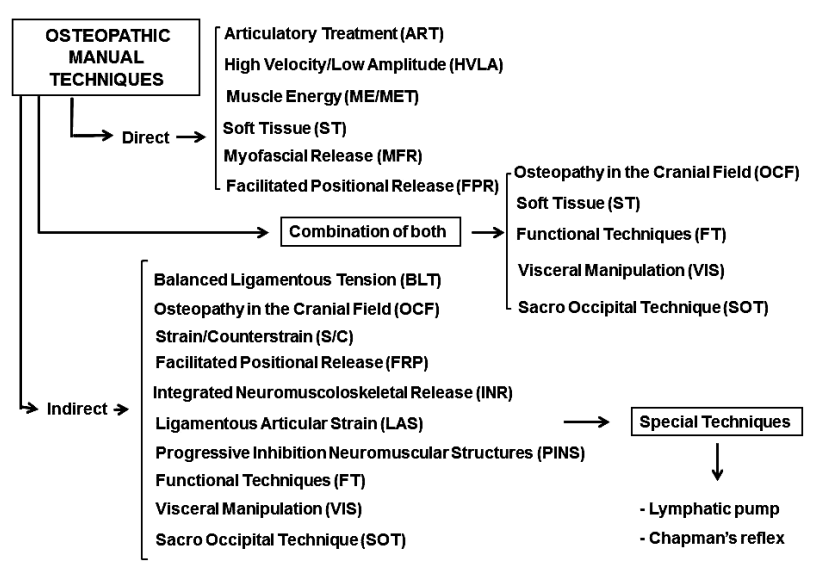

Figure 3. Overview of OMT techniques.

systems, spine, viscera, thoracic and pelvic diaphragms in order to restore homeostatic balance, normalize autonomic activity in the intestine, promote lymphatic flow 
and address SD. The first step assesses the pattern of signs and symptoms. Safety is paramount. If alarm signals, or "Red Flags" [21,22], are present the patient should be referred to a physician (Table 2).

Sagittal Plane Symmetry and the Common Compensatory Pattern (CCP) are observed in the postural examination $[23,24]$. The therapist then starts palpation using A.R.T.T. diagnostic criteria to search for SD. Attention focuses on sympathetic innervation from the lower thoracic to the upper lumbar spinal segments (T4-L2) via the collateral sympathetic ganglia (celiac, upper and lower mesenteric). Visceral afferent stimulation activates the hypothalamus pituitary adrenal axis and the autonomic nervous system, involving the release of neurotransmitters and hormones such as corticotropin-releasing factor which may play a role in modulating emotions [25]. Moving to parasympathetic innervation the therapist concentrates on the vagus nerve, which innervates the small intestine and colon up to the splenic flexure. Treatment of the upper cervical spine, cranial base and general sub-occipital area releases tension on the vagus nerve, as it passes through the jugular foramen. Assessment continues with the mid-cervicals $(\mathrm{C} 3,4,5)$, where the phrenic nerve arises to supply the thoracic diaphragm (Figure 4). Pelvic and sacroiliac joints should be treated as they may affect the pelvic splanchnic nerves (S2, 3, 4) which innervate the descending and sigmoid colon tracts $[26,27]$.

Table 2. Red Flags for IBS.

\begin{tabular}{|c|}
\hline Ulcerative Colitis and Crohn's Disease \\
\hline Diarrhea \\
\hline Constipation \\
\hline Fever \\
\hline Abdominal pain \\
\hline Rectal bleeding \\
\hline Night sweats \\
\hline \multirow{2}{*}{$\begin{array}{c}\text { Decreased appetite, nausea, weight loss } \\
\text { Skin lesions }\end{array}$} \\
\hline \\
\hline \multirow{2}{*}{ Uveitis (inflammation of the eye) } \\
\hline \\
\hline $\begin{array}{c}\text { Migratory arthralgias } \\
\text { Hip pain (iliopsoas abscess) }\end{array}$ \\
\hline Hip pain (iliopsoas abscess) \\
\hline Colorectal Cancer \\
\hline \multirow{4}{*}{$\begin{array}{l}\text { Rectal bleeding, haemorrhoids } \\
\text { Abdominal, pelvic, back, or sacral pain } \\
\text { Back pain that radiates down the legs } \\
\text { Changes in bowel patterns }\end{array}$} \\
\hline \\
\hline \\
\hline \\
\hline Advanced Stages \\
\hline $\begin{array}{l}\text { Constipation progressing to obstipation } \\
\text { Diarrhea with much mucus }\end{array}$ \\
\hline Nausea, vomiting \\
\hline Abdominal distention \\
\hline Weight loss \\
\hline Fatigue and dyspnea \\
\hline
\end{tabular}
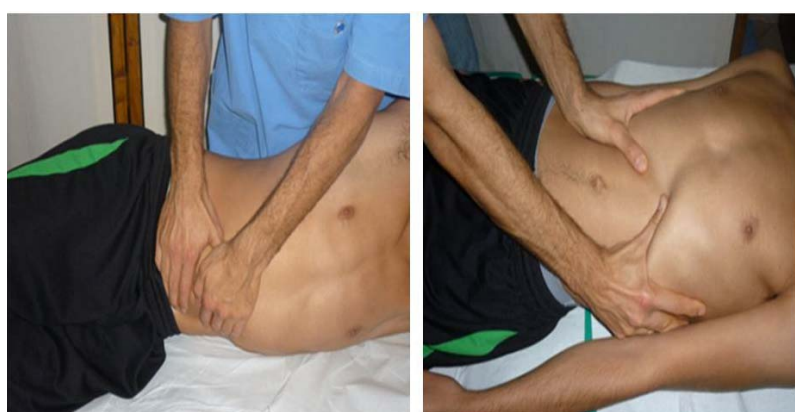

Figure 4. Combination of direct and indirect techniques.

In IBS, lymphatic and venous congestion should be treated by the lymphatic pump techniques (Figure 5) and stimulation of chapman's reflex points (Figure 6). They increase lymph flow and improve blood circulation thus facilitating healing and enhancing the efficacy of any medication $[28,29]$.

The OMT plan for IBS is shown in Figure 7.

\section{NUTRITION}

Hippocrates (460-357 BC) was the first to suggest that "He who does not know food, cannot understand the diseases of man". A. T. Still believed the body made its own remedies against disease and other toxic conditions when it was structurally normal and had favorable environmental conditions and adequate nutrition. In the modern diet increased consumption of high-density, lowquality foods, particularly when rich in refined starches, sugar, and lipids and poor in natural antioxidants and fibre, promote systemic inflammation which underlies chronic pain and several degenerative diseases [30,31].

The role of diet in IBS remains to be elucidated. Food could contribute to symptom onset or even cause IBS itself [32]. IBS symptoms are often exacerbated immediately after eating because of increased intraluminal volumes or motor activity, via excessive activation of vagal mechano- and chemo-receptors [5]. Food allergy (immune-mediated) or intolerance (not immune-mediated) could play a role in IBS [33]. Food antigens may result in low-grade mucosal inflammation and immune system activation through mast cell activation and degranulation, which in turn secrete several chemical messengers (e.g. transmitters, cytokines) that mediate bowel sensor-motor dysfunction [34]. Finally, some foods may alter gut microbes or "microbiota" qualitatively, thus increasing gas production. Transit of exogenous gas loads was impaired in patients with IBS and gas retention reproduced their bloating symptoms [35]. Proliferation of certain species that produce short-chain fatty acids and deconjugate bile acids more avidly could lead to clinically significant changes in water and electrolyte transport in the colon and affect colonic motility and/or sensitivity [32].

Furthermore, the microbiota greatly impacts on the 

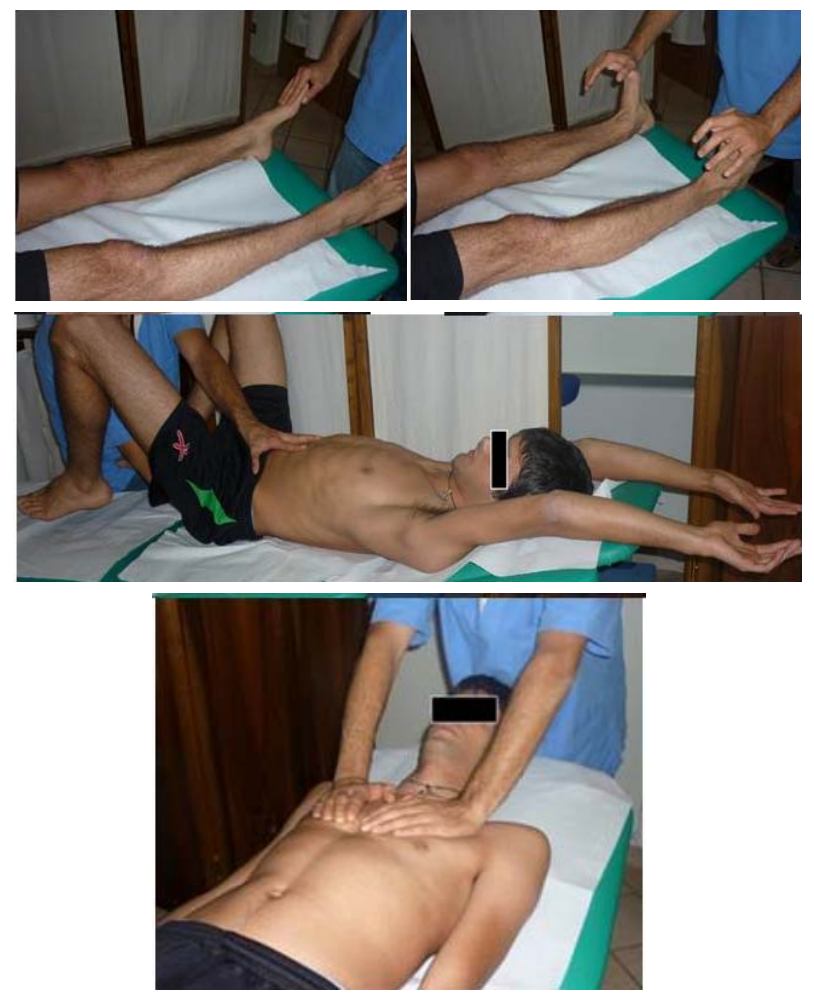

Figure 5. The lymphatic pump techniques.
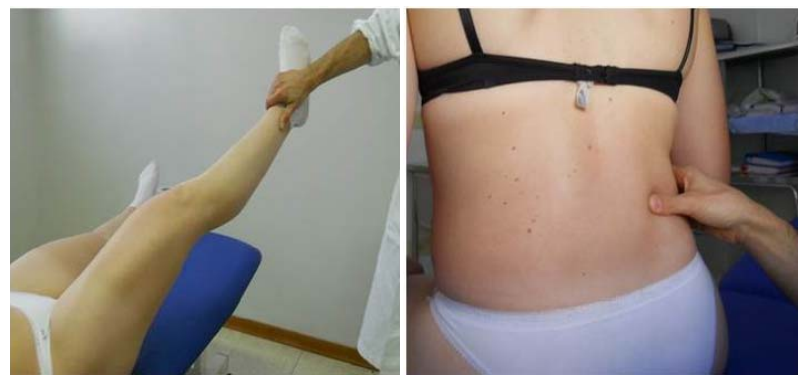

Figure 6. Stimulation of Chapman's reflex points.

bi-directional brain-gut axis communication [36]. Innovative research suggested microbiota affected the vagus nerve and modulated systemic levels of tryptophan, a serotonin precursor [37]. Together with the finding that dietary carbohydrates facilitated tryptophan entry into the brain, these data lead to the hypothesis that specific modulation of enteric microbiota and dietary habit is a useful strategy for GIT stress-related disorders, like IBS $[36,38]$.

\section{Nutrition for IBS}

Due to fear of symptom exacerbation patients with Care should be exercised milk, wheat and eggs which are often reported to trigger symptoms and over salycylate or amine-rich foods as they worsen symptoms [39]. Fatty food should be avoided since lipids strongly stimulate motor and sensory responses which are often increased in IBS patients [40]. Patients, particularly those with IBS-D, should be particularly careful with carbohydrates like lactose, fructose and sorbitol as they are malabsorbed.

Fibre supplements should be introduced into the diet very gradually so as to minimize bloating, particularly in patients with IBS-C [39]. Patients should receive more soluble than insoluble fibre [41].

Probiotic supplements aid intestinal pain management by increasing mucosal anti-inflammatory cytokines while reducing the pro-inflammatory, thus enhancing the mucosal barrier function [42-45].

A carbohydrate-rich, protein-poor meal increased brain concentration of tryptophan, and increased serotonin synthesis $[46,47]$. Given the importance of serotonin as a neurotransmitter in the ENS, one might speculate that a carbohydrate-rich diet might improve mood, alleviating anxiety, depression and somatisation.

Table 3 summarizes an appropriate nutritional approach for IBS patients.

\section{DISCUSSION}

In diseases like IBS which link the somatic and visceral systems, the gastrointestinal barrier impairment increases the risk of developing infectious, inflammatory and immune-mediated disorders. Recent research has focused on the role of the intestine in health maintenance and the "gut health" approach is providing a new strategy in preventive medicine [48]. IBS, however, often goes untreated, as approximately $70 \%$ of patients do not seek medical care and in many countries, including the US, prescription medication insurance coverage for IBS is decreasing [49]. Furthermore traditional medication offers marginal efficacy with only $7 \%-15 \%$ gains over placebo, and safety concerns are growing [43].

Table 3. Dietary recommendations.

\section{AVOID:}

Large meals,Alcoholic beverages, Caffeinated drinks, Carbonated drinks, Fatty food, Hot spices

\section{CAUTION:}

Eggs, Wheat products, High-salicylate foods: nuts, corn, wine, tomato, seasoned meat, ecc.; High-amine foods: chocolate, banana, avocado, spinach, ecc.; High-lactose-content products: milk, ice cream, yogurt; High-fructose-content products: honey, date, orange, apple, pear, corn syrup; Sorbitol-content products: artificial sweeteners, stone fruits; Gas-producing foods: beans, peas, broccoli, cabbage, bran.

\section{LOW INCREASE:}

Carbohydrate-rich foods: buckwheat, oats, etc.; Soluble fibre: oat bran, soy, barley, rice, currants, etc.

\section{ADD:}

Soluble fiber: psyllium or Ispaghula; Probiotic combinations of Lactobacilli, Bifidobacteria and Streptococci 


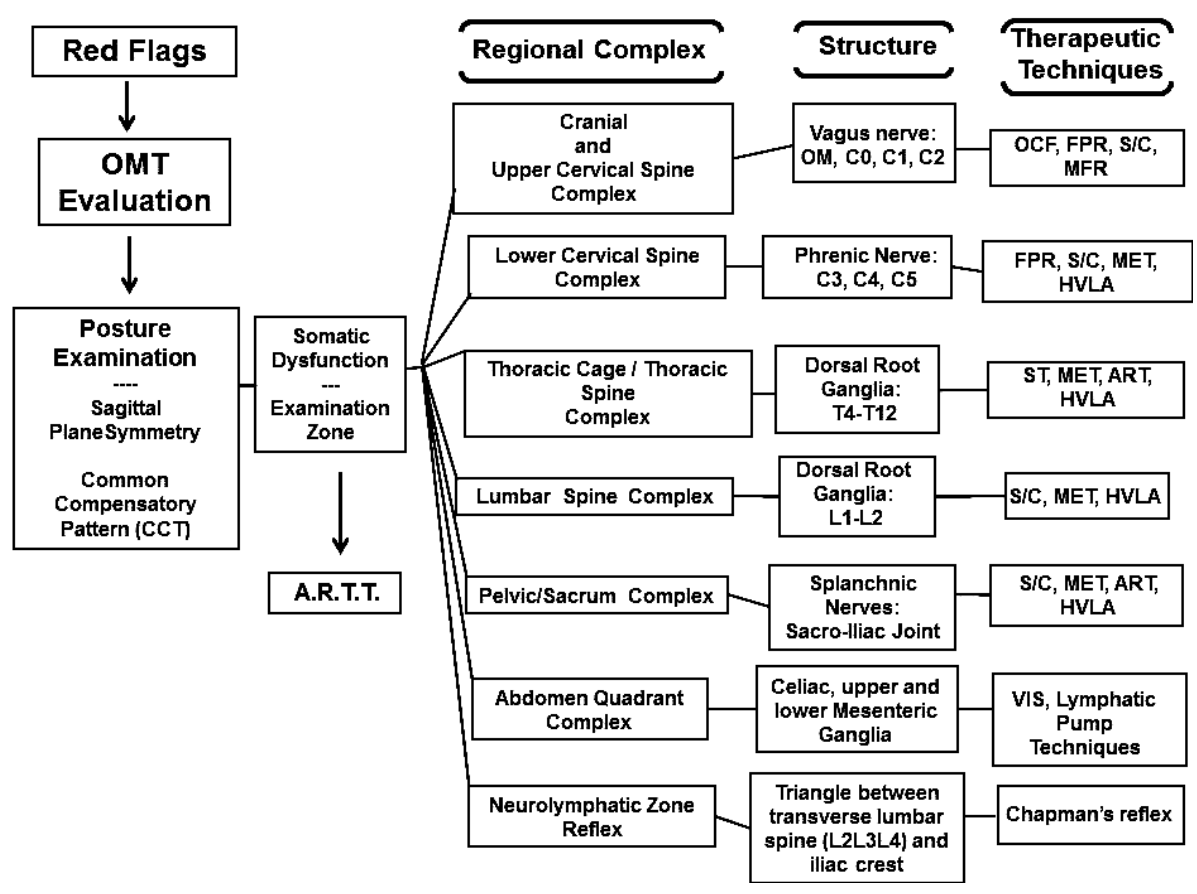

Figure 7. OMT plan for IBS.

In patients with IBS, the introduction of "direct access" (which allows patients to bypass the general practitioner in favour of a physical or manual therapist) is another major factor underlying their desire for more holistic and "natural" treatment options like OMT, dietary modification and nutritional supplementation. OMT provides the patient with relaxation, normalized autonomic control mechanisms, congestion and symptom relief, and better control over his/her reactions to stress. The dietary modifications proposed in this paper may remove triggers of IBS while the nutritional supplements help re-balance gut health.

\section{CONCLUSION}

Therapists now need to take a more holistic view of patients with IBS and provide a more comprehensive treatment model. Combining OMT with appropriate nutrition appears to be an optimal approach and might represent a promising strategy. Although this therapeutic plan may not be ideal for everyone, the majority of patients could well benefit from its use.

\section{ACKNOWLEDGEMENTS}

The authors would like to thank Dr Geraldine Anne Boyd for editing this paper.

\section{REFERENCES}

[1] Drossman, D.A., Corazziari, E., Delvaux, M., Spiller, R., Talley, N.J., Thompson, W.G., et al. (2006) Rome III: The functional gastrointestinal disorders. 3rd Edition, Degnon Associates, McLean, 885-893.

[2] El-Salhy, M. (2012) Irritable bowel syndrome: Diagnosis and pathogenesis. World Journal of Gastroenterology, 7, 5151-5163.

[3] Kennedy, P.J., Clarke, G., Quigley, E.M., Groeger, J.A., Dinan, T.G. and Cryan, J.F. (2012) Gut memories: Towards a cognitive neurobiology of irritable bowel syndrome. Neuroscience and Biobehavioral Reviews, 36, 310-340. doi:10.1016/i.neubiorev.2011.07.001

[4] Waring, W.S., Chui, M., Japp, A., Nicol, E.F. and Ford, M.J. (2004) Autonomic cardiovascular responses are impaired in women with irritable bowel syndrome. Journal of Clinical Gastroenterology, 38, 658-663. doi:10.1097/01.mcg.0000135362.35665.49

[5] Schmulson, M.J. (2001) Brain-gut interaction in irritable bowel syndrome: New findings of a multicomponent disease model. The Israel Medical Association Journal, 3, 104-110.

[6] Barbara, G., De Giorgio, R., Stanghellini, V., Cremon, C., Salvioli, B. and Corinaldesi, R. (2004) New pathophysiological mechanisms in irritable bowel syndrome. Alimentary Pharmacology and Therapeutics, 20, 1-9. doi:10.1111/j.1365-2036.2004.02036.x

[7] Karantanos, T., Markoutsaki, T., Gazuoli, M., Anagnou, N.P. and Karamanolis, D.G. (2010) Current insights into pathophysiology of Irritable Bowel Syndrome. Gut Pathogens, 2, 1-8. doi:10.1186/1757-4749-2-3

[8] Seminowicz, D.A., Labus, J.S., Bueller, J.A., Tillisch, K., Naliboff, B.D., Bushnell, M.C., et al. (2010) Regional grey matter density changes in brains of patients with irritable bowel syndrome. Gastroenterology, 139, 48-57. doi:10.1053/i.gastro.2010.03.049 
[9] Bashashati, M., Rezaei, N., Andrews, CN., Chen, CQ., Daryani, N.E., Sharkey, K.A., et al. (2012) Cytokines and irritable bowel syndrome: Where do we stand? Cytokine, 57, 201-209. doi:10.1016/j.cyto.2011.11.019

[10] Ford, A.C. and Talley, N.J. (2011) Mucosal inflammation as a potential etiological factor in irritable bowel syndrome: A systematic review. Journal of Gastroenterology, 46, 421-431. doi:10.1007/s00535-011-0379-9

[11] Clarke, G., Fitzgerald, P., Hennessy, A.A., Cassidy, E.M., Quigley, E.M.M., Ross, P., et al. (2010) Marked elevations in pro-inflammatory polyunsaturated fatty acid metabolites in females with irritable bowel syndrome. Journal of Lipid Research, 51, 1186-1192. doi:10.1194/jlr.P000695

[12] Lee, B.J. and Bak, Y.T. (2011) Irritable bowel syndrome, gut microbiota and probiotics. Journal of Neurogastroenterology and Motility, 17, 252-266. doi:10.5056/jnm.2011.17.3.252

[13] Elsenbruch, S. (2011) Abdominal pain in Irritable Bowel Syndrome: A review of putative psychological, neural and neuro-immune mechanism. Brain, Behaviour, and Immunity, 25, 386-394. doi:10.1016/j.bbi.2010.11.010

[14] Mayer, E.A. and Gebhart, G.F. (1994) Basic and clinical aspects of visceral hyperalgia. Gastroenterology, 107, 271-293.

[15] Hungin, A.P.S., Whorwell, P.J., Tack, J. and Mearin, F. (2003) The prevalence, patterns and impact of irritable bowel syndrome: an international survey of 40,000 subjects. Alimentary Pharmacology and Therapeutics, 17, 643-650. doi:10.1046/j.1365-2036.2003.01456.x

[16] Glover, J.C. (2006) Educational Council on Osteopathic Principles. Glossary of osteopathic terminology. American Association of Colleges of Osteopathic Medicine, Chicago. http://www.do-online.org/pdf/sir_collegegloss.pdf

[17] Nelson, K.E. (2007) Diagnosing somatic dysfunction. In: Nelson, K.E. and Glonek, T., Eds., Somatic Dysfunction in Osteopathic Family Medicine, Lippincott Williams \& Wilkins, 12-13.

[18] Chaitow, L. (2012) The ARTT of palpation? Journal of Bodywork \& Movement Therapies, 16, 129-131. doi:10.1016/j.jbmt.2012.01.018

[19] DiGiovanna, E.L. (2005) Goals, classifications, and models of osteopathic manipulation. In: DiGiovanna, E.L., Schiowitz, S. and Dowling, D.J., Eds., An Osteopathic Approach to Diagnosis and Treatmen, 3rd Edition, Lippincott Williams \& Wilkins, 77-79.

[20] SOPE (2010) The scope of osteopathic practice in Europe. European Federation of Osteopaths editors, Brussels.

[21] APTA (2011) Today's physical therapist: A comprehensive review of a 21 st-Century health care profession. American Physical Therapy Association

[22] Goodman, C.C. and Snyder, T.K. (2007) Differential diagnosis for physical therapists. Screening for referral. 4th Edition, Elsevier, Mosby Saunders, 393-395.

[23] Schiowitz, S. and Dowling, D.J. (2005) Structural Examination and Documentation. In: DiGiovanna, E.L., Schiowitz, S. and Dowling, D.J., Eds., An Osteopathic
Approach to Diagnosis and Treatment, 3rd Edition, Lippincott Williams \& Wilkins, 53-63.

[24] Parsons, J. and Marcer, N. (2005) Irritable bowel syndrome. In: Osteopathy-Models for diagnosis, treatment and practice, Elsevier, Churchill Livingstone, 265-269.

[25] O’Malley, D., Quigley, E.M.M., Dinan, T.G. and Cryan, J.F. (2011) Do interactions between stress and immune responses lead to symptom exacerbations in irritable bowel syndrome? Brain, Behavior, and Immunity, 25, 1333-1341. doi:10.1016/j.bbi.2011.04.009

[26] Stone, C.A. (2007) Visceral and obstetric osteopathy. Elsevier, Churchill Livingstone, 111-149.

[27] DeStefano, L. (2011) Greenman's principles of manual medicine. 4th Edition, Lippincott Williams \& Wilkins.

[28] Capobianco, J.D. (2005) Chapman's Reflex Points. In: DiGiovanna, E.L., Schiowitz, S. and Dowling, D.J. Ed., An Osteopathic Approach to Diagnosis and Treatment. 3rd. Lippincott Williams \& Wilkins, 113-117.

[29] Hodge, L.M. and Downey, H.F. (2011) Lymphatic pump treatment enhances the lymphatic and immune systems. Experimental Biology and Medicine, 236, 1109-1115. doi:10.1258/ebm.2011.011057

[30] Seaman, D.R. (2002) The diet-induced proinflammatory state: A cause of chronic pain and other degenerative diseases? Journal of Manipulative and Physiological Therapeutics, 25, 168-79. doi:10.1067/mmt.2002.122324

[31] Galland, L. (2010) Diet and inflammation. Nutrition in Clinical Practice, 25, 634-640. doi:10.1177/0884533610385703

[32] Morcos, A., Dinan, T. and Quigley, E.M.M. (2009) Irritable bowel syndrome: Role of food in pathogenesis and management. Journal of Digestive Diseases, 10, 237-246. doi:10.1111/j.1751-2980.2009.00392.x

[33] Katiraei, P. and Bultron, G. (2011) Need for a comprehensive medical approach to the neuroimmuno-gastroenterology of irritable bowel syndrome. World Journal of Gastroenterology, 17, 2791-2800.

[34] Park, M.I. and Camilleri, M. (2006) Is there a role of food allergy in irritable bowel syndrome and functional dyspepsia? A systematic review. Neurogastroenterology and Motility, 18, 595-607.

doi:10.1111/j.1365-2982.2005.00745.x

[35] Agrawal, A. and Whorwell, P.J. (2008) Review article: Abdominal bloating and distension in functional gastrointestinal disorders-Epidemiology and exploration of possible mechanisms. Alimentary Pharmacology \& Therapeutics, 27, 2-10. doi:10.1111/j.1365-2036.2007.03549.x

[36] Cryan, J.F. and O'Mahony, S.M. (2011) The microbiomegut-brain axis: From bowel to behavior. Neurogastroenterology and Motility, 23, 187-192. doi:10.1111/j.1365-2982.2010.01664.x

[37] Grenham, S., Clarke, G., Cryan, J.F. and Dinan, T.G. (2011) Brain-gut-microbe communication in health and disease. Frontiers in Physiology, 2, 94. doi:10.3389/fphys.2011.00094

[38] Fernstrom, J.D. and Wurtman, R.J. (1971) Brain sero- 
tonin content: Increase following ingestion of carbohydrate diet. Science, 174, 1023-1025. doi:10.1126/science.174.4013.1023

[39] Cabré, E. (2011) Clinical Nutrition University: Nutrition in the prevention and management of irritable bowel syndrome, constipation and diverticulosis. The European e-Journal of Clinical Nutrition and Metabolism, 6, e85e95.

[40] Simren, M., Abrahanmasson, H. and Bjornsson, E.S. (2007) Lipid induced colonic hypersensitivity in irritable bowel syndrome: The role of bowel habits, sex and psychological factors. Clinical Gastroenterology and Hepatology, 5, 201-208. doi:10.1016/j.cgh.2006.09.032

[41] Bijkerk, C.J., de Wit, N.J., Muris, J.W.M., Whorwell, P.J., Knottnerus, J.A. and Hoes, A.W. (2009) Soluble or insoluble fibre in irritable bowel syndrome in primary care? Randomised placebo controlled trial. British Medical Journal, 339, b3154. doi:10.1136/bmj.b3154

[42] Brenner, D.M., Moeller, M.J., Chey, W.D., Schoenfeld, P.S. (2009) The utility of probiotics in the treatment of irritable bowel syndrome: A systematic review. American Journal of Gastroenterology, 104, 1033-1049. doi:10.1038/ajg.2009.25

[43] Chey, W.D., Maneerattaporn, M. and Saad, R. (2011) Pharmacologic and complementary and alternative medicine therapies for Irritable Bowel Syndrome. Gut and Liver, 5, 253-266.

[44] O’Mahony, L., Mccarthy, J., Kelly, P., Hurley, G., Luo, F.,
Chen, K., et al. (2005) Lactobacillus and bifidobacterium in irritable bowel syndrome: Symptom responses and relationship to cytokine profiles. Gastroenterology, 128, 541-551. doi:10.1053/i.gastro.2004.11.050

[45] Ohland, C.L. and MacNaughton, W.K. (2010) Probiotic bacteria and intestinal epithelial barrier function. American Journal of Physiology-Gastrointestinal and Liver Physiology, 298, G807-G819. doi:10.1152/ajpgi.00243.2009

[46] Lyons, P.M. and Truswell, A.S. (1988) Serotonin precursor influenced by type of carbohydrate meal in healthy adults. American Journal of Clinical Nutrition, 47, 433439.

[47] Wurtman, R.J., Wurtman, J.J., Regan, M.M., McDermott, J.M., Tsay, R.H. and Breu, J.J. (2003) Effects of normal meals rich in carbohydrates or proteins on plasma tryptophan and tyrosine ratios. American Journal of Clinical Nutrition, 77, 128-132.

[48] Bischoff, S.C. (2011) "Gut health": A new objective in medicine? BMC Medicine, 9, 24. doi:10.1186/1741-7015-9-24

[49] Drossman, D.A., Camilleri, M., Mayer, E.A. and Whitehead, W.E. (2002) AGA technical review on irritable bowel syndrome. Gastroenterology, 123, 2108-2131 doi:10.1053/gast.2002.37095

[50] Maitland, G.D., Hengeveld, E., English, K. and Banks, K. (2005) Maitland's vertebral manipulation. 7th Edition, Butterworth Heinemann, Oxford. 